\title{
The simplicity and complexity, accuracy and fuzziness of Sport technology
}

\author{
Xiang yunping \\ Zhoukou Normal University,Henan,China,466000
}

Keywords: simplicity and complexity, accuracy and fuzziness

Abstract. With the improvement of tennis technology in China, it is necessary to study the composition of its technology philosophically. This article attempts to make a preliminary discussion on some aspects from the simplicity and complexity, accuracy and fuzziness of natural dialectics category of tennis technique, to improve the knowledge of the nature and law of tennis technology from the philosophical point of view , and also provide the philosophical basis for the improve the level of tennis.

\section{1、The acquaintance of these two categories}

The development of tennis is a process from simple to complex, continuous improvement and perfection. From the development of tennis technology, the process has been experienced from simple technology to complex technology, from single tactics to comprehensive and changeable tactics. From the theory of tennis movement, it not only has experienced from simple to complex, from fuzzy to precise, but also experienced the process of describing tennis movement from complexity to simplicity, from precision to vagueness.

In order to understand complex things, phenomena and processes, it is essential to break them down into simple elements or parts before they can be recognized. This is the simplicity principle. Because the elements of a complex system are not simple combinations, there are complex networks of interconnections and constraints. Therefore, you must be studied the integrity and complexity of the system. The formation of the dialectical process from simplicity to complexity, from complexity to simplicity combines the simple principle of knowing things and the complexity of the study of things. The tennis movement is very open and complex, the pitch shifting, the movement state of the athletes, the use of technique and tactics are followed the field situation and improvisation, but this movement is composed of simple and basic human movement. Tactics come down to technology, technology comes down to the movement of people and spheres in space. They are both simple and complex. They are dialectical unity of simplicity and complexity. In tennis match, the use of tactics is very simple, mainly is the defense, but technology is complicated, the different combination of a variety of difficult moves, especially in the doubles match tacit understanding cooperation constitutes the game fast, flexible and diverse, accurate intense scenes. This simple and complex case is often used in tennis movement. The understanding of objective things always experiences from fuzzy to precise, accurate qualitative accuracy said matters, the degree of fuzziness said things uncertain, it reflects to know about things "either/or" and "is also the essential" contradictory relations. Due to the complexity of tennis movement, the antagonistic and polytropy of the screen, it is impossible to evaluate it accurately, and only have fuzzy and approximate evaluation. For example, the score is a digital assessment of the technical and tactical effects of both parties. The conventional statistics of field technology are quantitative evaluation of technology. All of these is not absolutely accurate quantitative evaluation, just use fuzzy less accurate instead of more precise. Therefore, researching tennis should grasp the dialectical relationship between good accurate and fuzzy.

\section{2、The simplicity and complexity of tennis.}

Tennis technology can be divided into two parts: offensive technology and defensive technology also can be divided into 10 different basic technologies. According to various technology forms, it 
also can be divided into more than 30 specific technologies. For example, the batting methods, there are 13 different types of batting methods; the main points of the action and the motion state of the ball are same. It will be more complicated if you analyze the batting technique from different angles. This seems to complicate matters, in fact, the more the classification of tennis technology, the more complex, but the composition of the unit is very simple. Classification is to analyze the different characteristics of technology, to analyze the technology link and its mutual relations, namely the complex objects and the process is decomposed into simple elements and basic links to investigate analysis.

From the point of view of system theory, a shot is considered as a system, and the elements of the system play a role respectively. Including the action method, the flight path of the ball and the drop point of the ball. The action method is the main factor; it is connected with other elements, remains at different levels, and has different functions. Similar to forehand shots, other tennis techniques are composed of a variety of complex elements at different levels, creating a bewildering and complex technical movement. However, from the commonness of these complex technical movement still can see that the simplicity is the biological movement which athletes do sports in the threedimensional space and time limited role in the of the ground and sphere. Analyzing the tennis technology must be from simple to complex, gradually grasp every detail to deepen the degree of understanding. The description of tennis technology should be based on the principle of simplicity to generalize a wide range of technical forms. As Engels discussed Darwin said: "the body from a few simple form to what we see today is increasingly diversified and complicated shape until human development series, basically is identified; So not only has the possibility to illustrate the existing of organic natural products, but also provides the basis, to trace the prehistoric times of the human spirit, to trace the human spirit from the simple, without structure, but there is stimulation induced organism lowest plasma have to thinking of every stage of development of the brain at present. Without these prehistoric times, it can be still a miracle the thinking of the human brain is Existence. "(the 1971 edition of the natural dialectics, p.176) this passage deeply illustrates the evolution of the dialectical relationship between simplicity and complexity. The development of tennis is accompanied by simplicity and complexity. We not ignore it.

\section{3、 The accuracy and fuzziness of tennis technology.}

A good tennis player can do very well in a match. For example, hit the lower extremities and trunk and upper arm, forearm and wrist power order, size, direction and functional point force is under the domination neural coordination effect on the ball, the ball along a certain path to spin into the field. This suggests that the visual and muscle proprioceptive sensation of tennis balls is extremely accurate. However, so far, no one has been able to accurately measure the size and timing of the muscles in the movements of a tennis ball. The understanding of these factors is fuzzy and uncertain, so in order to describe other objective things, we must unify the accuracy and fuzziness syndrome, improving accuracy and reducing ambiguity. A lot of good tennis players feel that is right, that is, if you hit the ball in the sweet zone of the racket, you can hit the ball faster, and the strike line is perfect. In my opinion, this is the precision of vagueness, which indicates that people can explain accuracy from the fuzzy side, and can also use accurate mathematical methods to study and deal with the phenomenon of fuzziness. Precision lies in the vagueness, the vagueness are the premise. In the analysis of the tennis technology, it cannot imagine leaving the precise ambiguity, also cannot imagine the idea of leaving the vague precision.

In short, tennis is the unity of simplicity and complexity, accuracy and fuzziness. Tennis has experienced a development process from simple to complex, from fuzzy to precise and from complex to simple, from precision to vagueness. As other things, the analysis of tennis techniques requires to insist the principle of simplicity and complexity. Not only to analyze the single function of each element of tennis technique, but also to analyze the complicated network relationship 
between each factor and also to study the integrity and the complexity of technology. The accuracy of tennis technology is our goal, but the proper fuzzy is necessary, precise in the arms of fuzzy, fuzzy on the premise of accurate.

Using fuzzy set theory to solve Tennis technical problems is very useful, only the understanding of the dialectical relationship between accuracy and fuzziness is correct, we can develop the analysis and research in the unity of the tennis technology. The constant improvement in the accuracy of tennis technology is consistent with the increasing awareness of the accuracy of things.

\section{4、Conclusions}

This article attempts to make a preliminary discussion on some aspects from the simplicity and complexity, accuracy and fuzziness of natural dialectics category of tennis technique, to improve the knowledge of the nature and law of tennis technology from the philosophical point of view ,and also provide the philosophical basis for the improve the level.

\section{References:}

[1] Zhang Wenjian. Discussion on Tennis Serve Technique [J]. Journal of Anyang Normal University, 2004.6.

[2] Zhao Zhiyan. A Brief Discussion on Tennis Serve Technique. Guizhou Sports Science and Technology [J], 2006: 30-33.

[3] Liu Lu. Sports Biomechanics for Serving Tennis Well[J]. Beijing Sport University Master's Thesis, 1998.

[4] Xu Zhigang. Tennis Serve Technique and Its Teaching and Training Strategy [J]. Journal of Yellow River Conservancy Technical College, 200886. 